\title{
Recuperación y Reutilización de Componentes Químicos Provenientes de Pilas y/o Baterías por Vía Húmeda
}

\author{
PABLO BONILLA*, WILSON MUÑOZ, PATRICIA VELASCO, MILTON VILLACÍS, PAULINA CÁCERES, \\ MÓNICA MEDIAVILLA Y YADIRA MAZA \\ Facultad de Ciencias Químicas, Universidad Central del Ecuador, \\ Laboratorio de Coloideo química \\ *Correspondencia autor: pablo2us@yahoo.com
}

\section{Resumen}

El presente trabajo busca recuperar y reutilizar los componentes químicos provenientes de pilas y baterías secas, alcalinas y secundarias mediante métodos en su mayoría húmedos basados en procesos fisicoquímicos, cuantitativos y cualitativos de separación, así como métodos simples de fusión, para luego usar los mismos en procesos industriales y procesos químicos de laboratorios.

Palabras Clave: reutilización, pilas, recuperación, componentes químicos.

\section{Abstract}

This work attempt to recovery and reuse chemical compound from dry, alkaline and secondary cell and batteries by wet methods based in qualitative, quantitative and physical chemistry processes of separations in order to avoid dangerous wastes. In the same way this compounds will be used in industrial or chemical processes in laboratories.

Key words: reuse, batteries, recovery, chemical compounds.

\section{Introducción}

Partiendo del principio de que una pila y/o batería está formada por diversas sustancias químicas, las cuales una vez terminada su acción pueden ser útiles para otros fines; es posible recuperar estas sustancias y reutilizarlas o transformar las mismas en otras que existen en el mercado local las cuales son importadas y vendidas a costos elevados para usarlas en laboratorios químicos como reactivos para análisis o en industrias para sus procesos. Adicionalmente se evita que estos componentes de las pilas ingresen en el ambiente y lo contaminen ${ }^{[5][6]}$.

En estudios cualitativos y cuantitativos iniciales se determinó los porcentajes de los diversos componentes (estudio previo realizado en la Facultad de Ciencias Químicas, laboratorio OSP de la Universidad Central), se encontró que existe un mayor porcentaje de compuestos de manganeso seguido de compuestos de hierro, zinc, carbono como constituyentes principales y como secundarios, sales de amonio, potasio, litio, cobre, níquel, mercurio, cobalto, aluminio y estaño, tanto en pilas secas, alcalinas, pilas botón cuanto en pilas secundarias o recargables.

Existe una amplia variedad de baterías, las cuales pueden ser clasificadas de acuerdo a su diseño básico que determina la cantidad de electricidad. Algunas pilas llamadas primarias son aquellas que luego de cumplir un tiempo dejan de trabajar luego que sus compuestos químicos han terminado una serie de reacciones químicas irreversibles. 
A diferencia de las secundarias, que pueden ser recargadas debido a que cambiando el sentido de reacción este se vuelve reversible y adquiere la fuerza electromotriz inicial ${ }^{[4]}$.

También puede clasificarse las baterías de acuerdo al contenido químico (naturaleza del electrolito); muchas baterías primarias están formadas por sustancias a manera de pasta, estas son llamadas pilas secas y otras en las cuales se tiene sustancias en estado semisólido, son las Ilamadas pilas húmedas.

La pila seca (ver figural) está conformada por un ánodo de cinc que constituye el envoltorio de la misma. El cátodo ocupa una posición central formado por una varilla de carbón. El electrolito es una pasta formada por dióxido de manganeso $\left(\mathrm{MnO}_{2}\right)$ más carbono, cloruro de amonio $\left(\mathrm{NH}_{4} \mathrm{Cl}\right)$ más cloruro de cinc y agua.

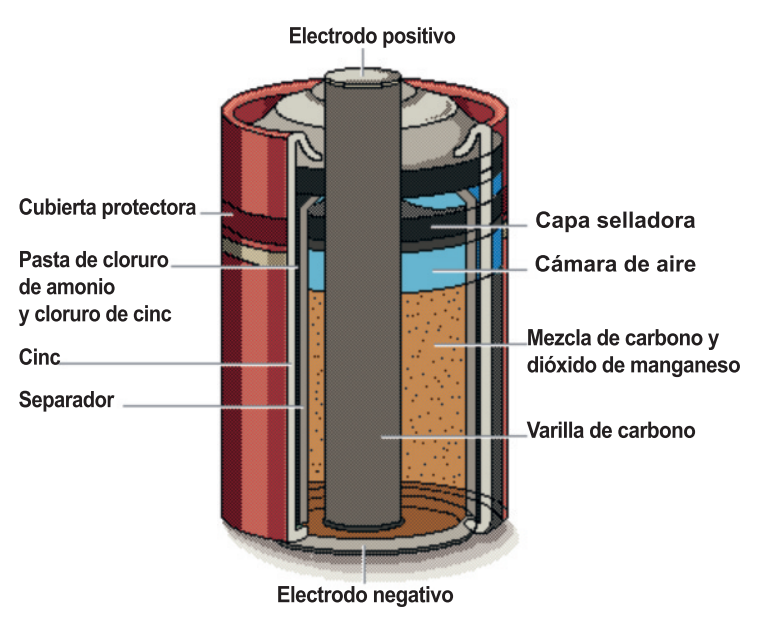

Figura 1. Esquema de una pila secal ${ }^{[8]}$

El sistema electroquímico de una pila seca está representado por el siguiente esquema ${ }^{[1]}$ :

$\mathrm{MnO}_{2}\left|\mathrm{NH}_{4} \mathrm{Cl}, \mathrm{aq}\right| \mathrm{Zn}$

Adicionalmente las reacciones que suceden en el electrodo positivo son:

$$
\mathrm{MnO}_{2}+\mathrm{H}_{2} \mathrm{O}+2 \mathrm{e}^{-} \rightarrow \mathrm{MnOOH}_{2} \mathrm{OH}^{-}
$$

Existen otras reacciones de termodinámica más compleja y que aproximadamente están representadas por las siguientes ecuaciones:

$$
\begin{aligned}
& \mathrm{Zn}+2 \mathrm{MnO}_{2}+2 \mathrm{H}_{2} \mathrm{O} \rightarrow \mathrm{Zn}(\mathrm{OH})_{2}+2 \mathrm{MnOOH} \\
& \mathrm{Zn}+2 \mathrm{MnO}_{2}+2 \mathrm{NH}_{4} \mathrm{Cl} \rightarrow \mathrm{Zn}\left(\mathrm{NH}_{3}\right)_{2} \mathrm{Cl}_{2}+2 \mathrm{MnOOH}
\end{aligned}
$$

En cuanto a las pilas alcalinas también tienen un ánodo de cinc; pero, pulverizado y amalgamado. El cátodo es un clavo central de acero. El electrolito es una pasta formada por $\mathrm{KOH}, \mathrm{MnO}_{2}$ y $\mathrm{H}_{2} \mathrm{O}$. Las semi-reacciones que tienen lugar son las siguientes ${ }^{[1]}$ :

$\mathrm{Zn}+4 \mathrm{OH}-\rightarrow \mathrm{ZnO}_{2}{ }^{2-}+2 \mathrm{H}_{2} \mathrm{O}+2 \mathrm{e}^{-}$

$$
\mathrm{Zn}+2 \mathrm{OH}-\rightarrow \mathrm{ZnO}+\mathrm{H}_{2} \mathrm{O}+2 \mathrm{e}^{-}
$$

Cabe anotar que las pilas secas funcionan a pH ácido y las alcalinas a pH básico y las pilas botón (ver figura 2) son similares a las pilas alcalinas; pero, el $\mathrm{MnO}_{2}$ se sustituye por $\mathrm{HgO} \circ \mathrm{Ag}_{2} \mathrm{O}$.

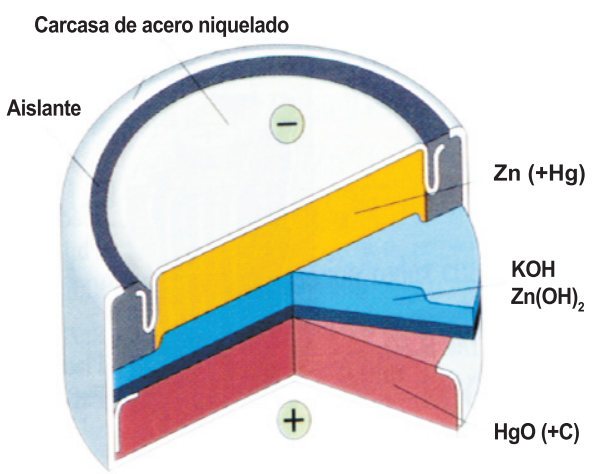

Figura 2. Esquema de pila botón ${ }^{[8]}$

En este caso las semi-reacciones catódicas son las siguientes:

$\mathrm{HgO}+\mathrm{H}_{2} \mathrm{O}+2 \mathrm{e}^{-} \rightarrow \mathrm{Hg}+2 \mathrm{OH}^{-}$

$\mathrm{Ag}_{2} \mathrm{O}+\mathrm{H}_{2} \mathrm{O}+2 \mathrm{e}^{-} \rightarrow 2 \mathrm{Ag}+2 \mathrm{OH}$

Por lo tanto, mediante reacciones específicas puede separarse los diversos componentes provenientes de la disolución acuosa de una mezcla de los mismos mediante marchas analíticas con lo que se van precipitando unos componentes y otros pasan al filtrado, y así al final obtener cada uno de los componentes químicos separados y listos para seguir procesos de purificación o directamente utilizados en nuevos procesos, como materia 
prima de los mismos ${ }^{[7]}$. Esto podemos complementar con lo dicho por Glynn, 1999[2] "Se dispone de diversos procesos físicos para la separación de sólidos y líquidos, entre ellos centrifugación, flotación, sedimentación y filtración".

\section{Método}

El proceso a seguir para la obtención, clasificación y tratamiento, una vez recolectadas las pilas, es clasificarlas en secas, alcalinas y recargables. A cada tipo de pilas se las separa de sus diversos componentes, tales como: recubrimientos plásticos, carcasas metálicas, pastas húmedas con electrolitos, electrodos sean metálicos o de carbono, cartón o plástico usados como aislante, tapas de hierro galvanizado.

Para los procesos de separación se usan aproximadamente 10 pilas secas de distintas marcas, 5 a 10 gramos de pilas botón y un promedio de 5 baterías de celular con un peso promedio de 23 gramos.

Las partes metálicas son separadas para una posterior fundición, las pastas húmedas y secas son sometidas a lavados, para luego ser tratados químicamente con ácidos minerales, para obtener extractos donde se determina y cuantifica por absorción atómica los diversos componentes (ver tabla 3 en resultados).

Adicionalmente a los procesos de tratamiento de muestras, también se realiza los procesos de separación y obtención de los diversos componentes químicos de las pilas, para lo cual a partir de muestras de 1 a 5 gramos de mezclas de sólidos pulverulentos, previamente lavados, se realiza digestiones con ácidos minerales diluidos con lo que se asegura la digestión total de materia orgánica e inorgánica oxidable.

Al realizar la digestión se obtiene disueltas tanto las sales ácidas solubles en medio acuoso, cuanto los componentes no solubles, los cuales son separados por filtración simple; de esta forma a la solución filtrada se la trata en medio básico para separar las diversas sustancias, según el pH de precipitación y sus respectivos Kps (producto de solubilidad) y obtenerlos como hidróxidos; la tabla 1 nos muestra que a un pH determinado y en una concentración determinada, que para el caso de los diversos componentes de la tabla, es de $1 \times 10^{-2} \mathrm{M}$ como concentración a pH inicial y $1 \times 10^{-6} \mathrm{M}$ para $\mathrm{pH}$ final, los diversos hidróxidos contenidos en una muestra pueden ir precipitando fraccionadamente.

Tabla 1. Valores de pH para precipitación de hidróxidos ${ }^{[3]}$

\begin{tabular}{|c|c|c|c|}
\hline Compuesto & Kps & pH inicial & pH final \\
\hline $\mathrm{Mg}(\mathrm{OH})_{2}$ & $6,1 \times 10^{-10}$ & 10,4 & 12,42 \\
\hline $\mathrm{Mn}(\mathrm{OH})_{2}$ & $4,5 \times 10^{-13}$ & 8,83 & 10,83 \\
\hline $\mathrm{Co}(\mathrm{OH})_{2}$ & $2 \times 10^{-15}$ & 7,65 & 9,65 \\
\hline $\mathrm{Ni}(\mathrm{OH})_{2}$ & $2 \times 10^{-15}$ & 7,65 & 9,65 \\
\hline $\mathrm{Zn}(\mathrm{OH})_{2}$ & $7 \times 10^{-18}$ & 6,43 & 8,43 \\
\hline $\mathrm{Cr}(\mathrm{OH})_{3}$ & $6 \times 10^{-31}$ & 4,25 & 5,90 \\
\hline $\mathrm{Al}(\mathrm{OH})_{3}$ & $1 \times 10^{-32}$ & 4,00 & 5,32 \\
\hline $\mathrm{Fe}(\mathrm{OH})_{3}$ & $1 \times 10^{-35}$ & 3,00 & 4,60 \\
\hline $\mathrm{Sn}(\mathrm{OH})_{2}$ & $6 \times 10^{-27}$ & 1,90 & 3,90 \\
\hline
\end{tabular}

En otras palabras, de la concentración de iones $\mathrm{OH}-$ depende que el hidróxido precipite o no. Mientras más soluble sea el hidróxido mayor será la concentración de los iones $\mathrm{OH}$ - necesarios para sobrepasar su producto de solubilidad; es decir, tanto menor deberá ser el valor del $\mathrm{pH}$ para que comience esta precipitación ${ }^{[3]}$.

A partir del producto de solubilidad, puede calcularse el valor de $\mathrm{pH}$ en que empieza y termina la precipitación de un hidróxido y por esta misma razón al tener una mezcla de componentes químicos con diversos metales, como es el caso de las pilas, podemos precipitar los mismos para separarlos de los demás sólo variando la concentración de hidróxido y midiendo el pH; ver tabla 2. 
Tabla 2. Potencial hidrógeno $(\mathrm{pH})$ de precipitación de diversos hidróxidos de acuerdo con la cantidad de $\mathrm{NaOH}$ añadido a soluciones digeridas de componentes sólidos de las pilas (datos obtenidos del estudio).

\begin{tabular}{|c|c|c|c|c|}
\hline Tipo de pila & pH precipitación & $\begin{array}{c}\text { Peso } \mathrm{NaOH} \\
\text { (gramos) }\end{array}$ & Color de precipitado & Tipo de hidróxido \\
\hline Secas y alcalinas & $\begin{array}{l}\mathrm{pH} i=2,06 \\
\mathrm{pH} f=12,71\end{array}$ & $\begin{array}{l}W i=6,26 \\
W f=7,26\end{array}$ & Pardo & $\begin{array}{c}\text { De hierro }(3+), \text { cinc y } \\
\text { manganeso }\end{array}$ \\
\hline \multirow{3}{*}{$\underline{\text { Pasta celeste }}$} & $\mathrm{pH} i=5,50$ & Wi= ------- & & De cinc y hierro $(3+)$ \\
\hline & $p H f=13,70$ & $W f=0,32$ & $\begin{array}{c}\text { Blanco y ligeramente } \\
\text { pardo } \\
\text { Pardo }\end{array}$ & \multirow[t]{2}{*}{$\begin{array}{c}\text { Plata, manganeso y } \\
\text { mercurio }\end{array}$} \\
\hline & $\begin{array}{l}\mathrm{pH} i=1,17 \\
\mathrm{pH} f=6,00 \\
\mathrm{pH} f=12,80\end{array}$ & $\begin{array}{c}W i=1,45 \\
W m=2,27 \\
W f=2,68\end{array}$ & $\begin{array}{l}\text { Negro } \\
\text { Negro }\end{array}$ & \\
\hline \multicolumn{5}{|l|}{ Recargables } \\
\hline \multirow[t]{3}{*}{$\underline{\text { Sólidos negros }}$} & $\begin{array}{l}\mathrm{pH} i=1,00 \\
\mathrm{pH} f=12,61\end{array}$ & $\begin{array}{l}W i=1,84 \\
W f=2,02\end{array}$ & Celeste verdoso & Cobre y níquel \\
\hline & $\mathrm{pH} \mathrm{m}=3,61$ & $W m=1,46$ & Lila, gris, azul y pardo & \\
\hline & $\begin{array}{l}\mathrm{pH} \mathrm{m}=6,20 \\
\mathrm{pH} f=12,50\end{array}$ & $\begin{array}{l}W m=2,18 \\
W f=2,20\end{array}$ & & Cobalto \\
\hline
\end{tabular}

\section{Resultados}

A continuación se detalla los componentes encontrados por absorción atómica en los diversos tipos de pilas.

Tabla 3. Resultados promedio de análisis de absorción atómica para los diversos tipos de pila.

\begin{tabular}{|c|c|c|c|c|c|c|}
\hline Tipo de pila & $\begin{array}{c}\mathrm{Hg} \\
\mathrm{mg} / \mathrm{l}\end{array}$ & $\begin{array}{c}\mathrm{Fe} \\
\mathrm{mg} / \mathrm{l}\end{array}$ & $\begin{array}{c}\mathrm{Ag} \\
\mathrm{mg} / \mathrm{l}\end{array}$ & $\begin{array}{c}\mathrm{Zn} \\
\mathrm{mg} / \mathrm{l}\end{array}$ & $\begin{array}{c}\mathrm{Mn} \\
\mathrm{mg} / \mathrm{l}\end{array}$ & $\begin{array}{c}\mathrm{Ni} \\
\mathrm{mg} / \mathrm{l}\end{array}$ \\
\hline Secas & 0,21 & 12,50 & $<1$ & 500 & $>10000$ & n.a \\
\hline Alcalinas & 0,07 & 8 & $<1$ & $>500$ & $>10000$ & n.a \\
\hline Botón & $\begin{array}{c}0,010- \\
0,100\end{array}$ & $1-3$ & $10-250$ & $>100$ & $>10000$ & n.a \\
\hline Recargables & $<0,250$ & $<2000$ & $<1$ & 360 & n.a & 1500 \\
\hline
\end{tabular}


Todos estos datos fueron obtenidos al tratar sólo las partes internas de las pilas; es decir, los sólidos pulverulentos y pastosos. En la tabla 4 se resume el porcentaje: peso /peso que representan estos componentes en el total de las pilas, así como los porcentajes de los otros componentes metálicos de carcasas y cubiertas.

Tabla 4. Porcentaje promedio peso-peso de los diversos elementos que componen las pilas.

\begin{tabular}{cccccc}
\hline Tipo de pila & $\begin{array}{c}\text { \% Pastas } \\
\text { Sólidos }\end{array}$ & \% Cubierta & \% Carbono & \% Metal puro & \% Electrolito \\
\hline Secas & 40 & 20 & 10 & 25 & $<5$ \\
Alcalinas & 40 & 40 & 15 & 3 & $<5$ \\
Botón & 35 & 48 & 8 & 4 & $<5$ \\
& 25 & 17 & 20 & 3 (aluminio) & $\begin{array}{c}\text { dieléctrico } \\
\text { Recargables }\end{array}$ \\
& & & & & \\
\hline
\end{tabular}

En base a los resultados preliminares (tablas 3 y 4 ) puede establecerse los rendimientos de los diversos componentes de las pilas, según su tipo como consta en las tablas $5,6,7$ y 8 .

Tabla 5. Rendimiento de compuestos químicos por tonelada de pilas secas.

\begin{tabular}{ccccc}
\hline Componentes & Manganeso & Zinc & Carbono & $\begin{array}{c}\text { Hierro } \\
\text { galvanizado }\end{array}$ \\
\hline kg./ton. pilas & 600 & 200 & 100 & 200 \\
\hline
\end{tabular}

Tabla 6. Rendimiento de compuestos químicos por tonelada de pilas alcalinas.

\begin{tabular}{ccccc}
\hline Componentes & Manganeso & Zinc & Carbono & $\begin{array}{c}\text { Hierro } \\
\text { galvanizado }\end{array}$ \\
\hline kg./ton. pilas & 600 & 200 & 150 & 400 \\
\hline
\end{tabular}

Tabla 7. Rendimiento de compuestos químicos de pilas botón.

\begin{tabular}{cccccc}
\hline Componentes & Manganeso & Zinc & Carbono & $\begin{array}{c}\text { Hierro } \\
\text { galvanizado }\end{array}$ & $\begin{array}{c}\text { Hierro } \\
\text { galvanizado }\end{array}$ \\
\hline kg./ton. pilas & 600 & 0,1 & 80 & 480 & 0,25 \\
\hline
\end{tabular}

Tabla 8. Rendimiento de compuestos químicos de pilas recargables.

\begin{tabular}{cccccc}
\hline Componentes & Cobalto & Cobre & Carbono & Níquel & Aluminio \\
\hline kg./ton. pilas & - & 60 & 450 & 1,5 & 200 \\
\hline
\end{tabular}




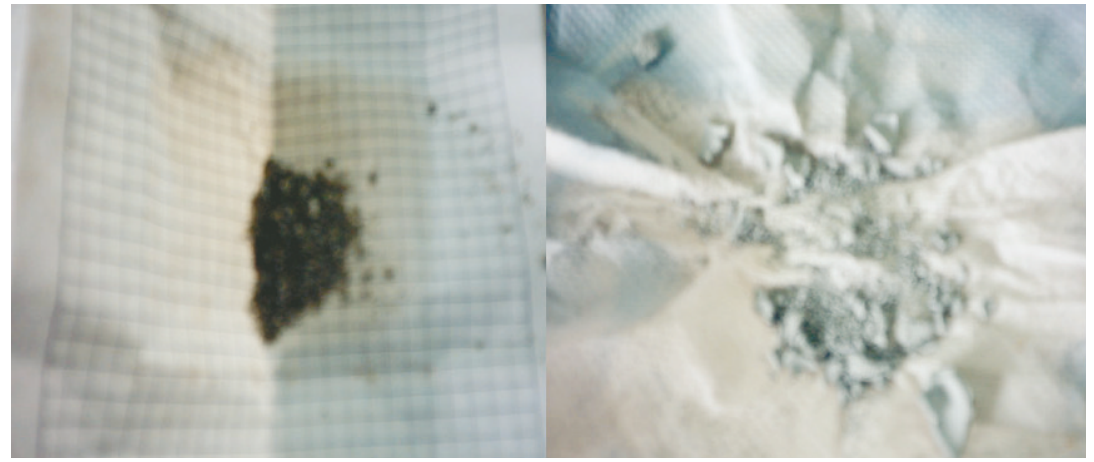

Figura 3. Sales de manganeso y cinc provenientes de pilas secas y alcalinas.

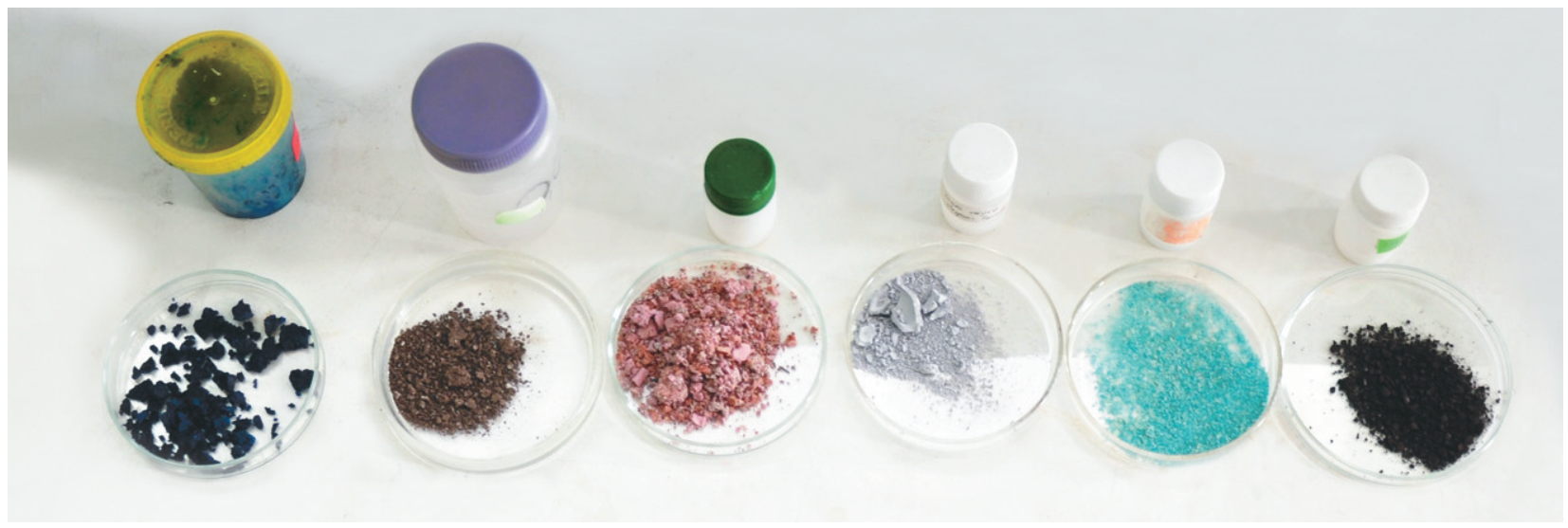

Figura 4. Compuestos de cobalto, manganeso y cobre obtenidos de las pilas recargables o de celular.

\section{Conclusiones}

Por tanto puede concluirse que controlando adecuadamente el proceso de digestión, puede separarse componentes insolubles de aquellos solubles de una mezcla de componentes de pilas, para luego mediante un seguimiento adecuado del $\mathrm{pH}$ ir precipitando de forma fraccionada el compuesto solubilizado y de esta forma obtener las diversas sustancias químicas que las componen. Además podemos de esta forma producir o proveer de sustancias químicas básicas para industria y laboratorios del país, la mayoría de las cuales son importadas y sus costos elevados.

\section{Agradecimientos}

Queremos dejar constancia de agradecimiento al personal del laboratorio OSP de la Facultad de Ciencias Químicas de la Universidad Central, por colaborar con el análisis de absorción atómica. También agradecemos a las autoridades de la Facultad por permitirnos realizar este trabajo en los laboratorios de la misma.

\section{Referencias}

1. Bagotsky V.S. Fundamentals of Electrochemistry, 2nd ed.; John Wiley \& Sons. Inc., Hoboken New Jersey, 2005; pp 350-353.

2. Glynn,H, Ingeniería Ambiental, segunda edición ,Ed. Prentice Hall, 1999,pp 645-654.

3. León, C, Química Analítica Cualitativa, 1ra.ed., 1984,pp 61-64,144,164.

4. http:/www. wasteonline.org.uk/resources/Information Sheets/Batteries.htm

5. http:://www.accurec.de/englisch/leistungen.html

6. http:// www.epa.gov

7. http://www.batteryuniversity.com/partone-20.htm

8. http://www.arbolesymedioambiente.es/Imagenes/pilas3.gif 